\title{
Minutes of the 36th General Assembly of the European Association for the Study of Diabetes
}

\section{Held in Hall A of the Jerusalem International Convention centre on Wednesday $20^{\text {th }}$ September 2000}

\author{
Present: Drs. J.Nerup (President) \\ R. Heine (Honorary Treasurer) \\ W. Waldhäusl (Editor-in-Chief, Diabetologia) \\ V.Jörgens (Executive Director) \\ and 94 members
}

The President welcomed everyone to the General Assembly and thanked Dr Segal for having organised such a wonderful Annual Meeting. The attendance had been close to 8.500 which was far beyond expectations.

\section{MINUTES, $35^{\text {th }}$ GENERAL ASSEMBLY 1999}

Since there were no comments, the minutes were approved and officially signed as a correct record.

\section{REPORTS}

\section{a) President}

Dr Nerup reported that the official programme of the Annual Meeting had had to be changed. The Study Group Meeting which was planned in collaboration with Palestinian colleagues to be held in Ramallah had to be re-located to the ICC in Jerusalem. One week before the Meeting a ban had been placed by the Palestinian authorities on Palestinian colleagues taking part in meetings in Ramallah and Jericho. They stated the ban would be lifted if part of the programme took place in East Jerusalem. Since this was seen to be political pressure, it was decided by the Executive Committee to cancel the meeting in Ramallah, which had been organised in close co-operation with the Palestinian Ministry of health and Palestinian colleagues. Dr Nerup reiterated that Palestinian colleagues were always welcome to attend any EASD activity and he was pleased to announce that Jordanian colleagues had joined the Postgraduate Meeting in Jerusalem.

Dr Nerup spoke about the partnership with the Juvenile Diabetes Foundation International (JDFI) and Novo Nordisk for a European Diabetes Research Programme. It was planned to make 12 million Euro available over the next 3 years. All three partners would consult for Type 1 and EASD and Novo Nordisk for Type 2. He encouraged everyone to apply for grants of 100.000 Euro per annum. He continued that it was the aim of the Executive Committee to make the European Foundation for the Study of Diabetes the major funding agency in Europe. In the USA 1 billion is spent annually on diabetes research, whereas in Europe no funds were earmarked for this. Very few national governments have a diabetes research programme which means that less than $1 / 10$ of USA funding is available in Europe.
Dr Nerup said he would be glad to receive ideas from the membership. He felt it was very important to stress that these new fund-raising activities would not interfere with national activities already in place.

Dr Nerup gratefully thanked the pharmaceutical industry for their support for the awards and fellowships:

The Claude Bernard sponsored by the Paul Neumann Association.

The Minkowski Lecture supported by Aventis.

The Camillo Golgi Lecture supported by the Castelli Pedroli family.

The EASD/Eli Lilly Award and the Scientists Training Course supported by Eli Lilly

EASD/Glaxo Wellcome Burden of Diabetes Research Fellowship and the Abstract Volume supported by Glaxo Wellcome. The EASD/Merck Travel Fellowships supported by Merck.

The EASD/Sankyo Insulin resistance Project Award supported by Sankyo.

The EASD/Amylin - Paul Langerhans Research fellowship supported by Amylin.

The Solidarity Fund supported by Novo Nordisk.

Dr Nerup expressed his special thanks to these pharmaceutical companies.

Dr Nerup announced the venues for future Annual Meetings which had been decided by the Executive Committee:

\section{Copenhagen/Malmö}

2007 Amsterdam

Dr Nerup brought his report to a close by thanking Council Members and Executive Committee Members for their support.

b) Honorary Treasurer:

Dr Heine opened his report by saying it was the second one to be given in Euro. He commented that the income from membership had remained fairly stable and that there had been no noticeable increase in income in 1997 due to the fact that there was no EASD Annual Meeting in that year. Income had, on the other hand, risen in 1998 and 1999 due to the input from the Annual Meetings of those years. He pointed out that interest rates had remained fairly stable. Regarding expenditure the amount spent on salaries had increased due to the hiring of an additional secretary. Expenditure had also increased substantially in 1999 due to the fact that an invoice from the publisher, Springer-Verlag, which should have been received in 1998, was only received in 1999.

Since Dr Heine's term of office as Honorary Treasurer had come to an end he thanked all the officers he had worked with and the secretariat in Düsseldorf. $\mathrm{He}$ wished his successor good luck.

Dr Nerup thanked the Honorary Treasurer for his report. No questions arose from the audience. The Honorary Auditors, Drs Landgraf and Skrha, confirmed the correctness of 
the accounting and the General Assembly approved the accounts with a unanimous vote of confidence.

\section{c) Honorary Secretary}

Dr Porta opened his report by explaining that all abstracts were reviewed anonymously by 35 experts, 14 of whom had gathered in May to put the programme together. Of $1640 \mathrm{ab}-$ stracts received 1127 had been accepted, 308 of these as orals. He stressed that there was no divide between orals and posters. $\mathrm{He}$ felt that the noise level (from air-conditioning, people ) was still too high in the poster area and that even more attention should be paid to this the following year. However the poster sessions had been well-attended and those involved seemed satisfied. The expenditure on Travel and Stayment grants had been increased and he felt that this money, roughly 100.000 Euro, had been well spent.

Dr Porta continued that the 15 officially recognised Study Groups were an essential component of the scientific life of the Association. Every year during the Annual Meeting there is a meeting with the Study Groups, some of which were more active than others. They are all represented on the website of the Association. Each year three Study Groups (working in rotation) presented at the Annual Meeting. He explained that the EDEG would present again the following year due to the fact that their meeting had to be re-located from Ramallah to Jerusalem in 2000.

Dr Porta said that putting together the scientific programme for the Annual Meeting was a considerable task which required the assistance of the membership. He therefore put out a plea for help, suggestions and information on new data available in order to produce an even better programme.

Dr Nerup thanked Dr Porta for the tremendous amount of work and time he had put into the scientific programme and asked if there were any questions.

Dr Korec said that he was unhappy with the way the abstracts were reviewed. His abstract had not been accepted, although he felt his results were better than some of those presented at the Annual Meeting; he felt even non-accepted abstracts should be mentioned by title. Dr Porta reiterated the fact that all abstracts were reviewed anonymously and that there was no question of any personal feelings regarding the decisions taken. He admitted good research might be turned down and dubious results accepted, but since the review committee changed every year this was kept to a minimum. Dr Nerup added that the Executive Committee had discussed the matter and it had been decided to maintain the present policy of not printing the titles of rejected abstracts. Dr Wollheim then offered his congratulations on the scientific content and pointed out that the Meeting had been well-attended even in a city like Jerusalem with its many tourist attractions.

\section{d) Editor-in-Chief, Diabetologia}

Dr Waldhäusl reported that the journal was doing well, with roughly 1.000 manuscripts a year being received. The acceptance rate is about $20 \%$, time from receipt to decision 50 days and mean publishing time from receipt to publication 191 days, which was down from 260. The Impact Factor had oscillated between 5 and 5.17. He continued that most manuscripts had been received from Japan, USA and the U.K and $50 \%$ of published manuscripts came from these countries with Sweden in $4^{\text {th }}$ place. He said there was also the option of putting the manuscripts on the internet once they had gone through the review process, which would speed up the mean publication time even more.

Dr Nerup thanked Dr Waldhäusl for the time and effort he spent on editing Diabetologia and asked if there were any questions to his report. Dr Halban said he felt that consider- able changes in phraseology were made during the editing of papers and he questioned if the changes were not too much, leading to a loss of individuality. Dr Waldhäusl replied that this was a sensitive issue and that the aim was to convey the message as clearly as possible.

e) Chair, Postgraduate Education Sub-Committee

Dr Boulton reported on the Courses which had already been held in the Kaunus, at Loipersdorf (organised by Dr T Pieber) and Lille (organised by Drs M. Hod from Israel and P. Fontaine from France). The latter was not only the first EASD Postgraduate course to be held in France, it was also the first course specifically on diabetes in pregnancy. Dr Boulton explained that the course planned to be held in Jericho after the Annual Meeting would now take place in Jerusalem and he was delighted that some Jordanian colleagues would attend. In 2000 there were also courses planned to take place in Italy (co-organised by the Chairman of the PGESC and Dr Fedele of Italy) and in Kluj (organised by Dr Hancu of Romania). For 2001 another course would be organised in Loipersdorf and discussions were underway for a course in Eastern Europe. In September 2001, directly after the Annual Meeting in Glasgow, a course would be planned in Ireland.

Dr Nerup thanked Dr Boulton for his report and when there were no questions he moved on to the next point.

\section{ELECTIONS}

a) President 2001-2004

The Council's election of Dr P Halban as successor to Dr Nerup was unanimously approved.

b) Vice-President 2000-2003

The Council's election of Dr A De Leiva was unanimously approved.

c) Honorary Secretary-Adjunct 2000-2001

The Council's election of Dr AJM Boulton was unanimously approved.

d) Dr Waldhäusl's term of office as Editor-in-Chief of Diabetologia was extended by two years to cover the transition period when the journal would be moved to Düsseldorf.

e) Council members 2001-2004: Drs U Di Mario (I), N D Hancu (Romania), J A Maassen (NL) and E Standl (D) were unanimously elected by the General Assembly to replace retiring members Drs T Halmos (H), M Hod (Israel), D Koev (Bulgaria) and I Valverdi (Spain).

Dr Nerup thanked all retiring officers for their successful collaboration.

\section{HONORARY MEMBERSHIP}

The nomination of Dr D Steiner (USA) for Honorary Membership was unanimously accepted by the General Assembly. Dr Steiner immediately expressed his thanks for this honour.

\section{ANY OTHER BUSINESS}

When there was no other business Dr Nerup thanked the officers and the secretariat for their efficiency and kindness and brought the meeting to a close. 
Table 1. Consolidated accounts for the year ended 31.December 1999

\begin{tabular}{|c|c|c|}
\hline & $\begin{array}{l}\text { Pound } \\
\text { Sterling }\end{array}$ & Euro \\
\hline Income & 1998 & 1999 \\
\hline Membership fees incl. Diabetologia & 306.028 & $439.983,75$ \\
\hline Donations & 202.464 & $288.256,13$ \\
\hline Annual Meeting Surplus & - & $628.656,20$ \\
\hline - repaid free loan & - & $75.895,57$ \\
\hline - reimbursed AM expenses & _ & $302.977,99$ \\
\hline Bank interest & 36.531 & $32.727,29$ \\
\hline Scientists Training Course Fees & 4.060 & $3.873,07$ \\
\hline Expired Bonds, taxes & & $135.190,68$ \\
\hline Total Income & 549.083 & $1.907 .560,68$ \\
\hline Expenditure & 1998 & 1999 \\
\hline Salaries (Secr, ExDir, Tax, Soc Ins) & 96.899 & $183.771,64$ \\
\hline Rent, Heating, Electricity & 9.791 & $14.282,69$ \\
\hline Post, Phone/Fax, Printing & 22.248 & $31.552,26$ \\
\hline Travel/Meeting Expenses & 51.790 & $58.262,56$ \\
\hline Office Costs, incl. Bank charges & 25.940 & $37.089,63$ \\
\hline Extra office help & 3.634 & $3.601,54$ \\
\hline Legal cost and accounting & 611 & $7.519,30$ \\
\hline Annual Meeting expenses & 76.176 & $125.303,54$ \\
\hline Abstract Volume & - & $15.338,76$ \\
\hline Free loans to Local Organisers & 51.471 & $151.791,14$ \\
\hline Travel and Stayment Grants & 63.587 & $82.842,88$ \\
\hline Prizes and Fellowships & 94.143 & $134.906,61$ \\
\hline Donation others & 5.000 & $7.589,56$ \\
\hline Diabetologia subscriptions & 91.243 & $508.858,49$ \\
\hline Membership Directory & 16.020 & - \\
\hline Editorial Office & 69.165 & $106.334,83$ \\
\hline Other items incl. PGESC & 7.535 & $16.088,54$ \\
\hline Scientists Training Course & 36.962 & $49.139,09$ \\
\hline EFSD & & $74.876,69$ \\
\hline Total & 722.216 & $1.609 .149,75$ \\
\hline
\end{tabular}

Exchange rates:

1998: f/Euro 0,6779; DM/Euro 1,95583

1999: $f /$ Euro 0,6588; DM/Euro 1,95583
All EASD Accounts

\begin{tabular}{|c|c|c|}
\hline & 31.12 .1998 & 13.12 .1999 \\
\hline Deutsch Mark & $\begin{array}{l}\text { Pound } \\
\text { Sterling }\end{array}$ & Euro \\
\hline $\begin{array}{l}\text { Current a/c } \\
\text { Fixed } \\
\text { Extra Savings } \\
\text { Bonds }\end{array}$ & $\begin{array}{r}28.932,84 \\
0,00 \\
52.985,75 \\
272.564,55\end{array}$ & $\begin{array}{r}111.097,95 \\
0,00 \\
79.471,22 \\
255.901,59\end{array}$ \\
\hline DM-Subtotal & $354.483,14$ & $446.470,77$ \\
\hline Pound Sterling & $\begin{array}{l}\text { Pound } \\
\text { Sterling }\end{array}$ & Euro \\
\hline $\begin{array}{l}\text { Current a/c } \\
\text { Business Res. a/c } \\
\text { Capital Res. A/c } \\
\text { Fixed }\end{array}$ & $\begin{array}{r}500,00 \\
204.259,82 \\
5.326,65 \\
120.000,00\end{array}$ & $\begin{array}{r}805,30 \\
362.086,38 \\
8.847,32 \\
644.240,00\end{array}$ \\
\hline Pound-Subtotal & $330.086,47$ & $1.015 .978,99$ \\
\hline Swiss Franc & $\begin{array}{l}\text { Pound } \\
\text { Sterling }\end{array}$ & Euro \\
\hline SFr Current + Fixed & $51.679,94$ & $85.914,58$ \\
\hline Spanish Pesetas & $\begin{array}{l}\text { Pound } \\
\text { Sterling }\end{array}$ & Euro \\
\hline Current a/c & 313,60 & $423,74^{\circ}$ \\
\hline Austrian Schilling & $\begin{array}{l}\text { Pound } \\
\text { Sterling }\end{array}$ & Euro \\
\hline Current a/c & $1.856,80$ & $-1-$ \\
\hline Total: & $738.419,95$ & $1.548 .788,08$ \\
\hline $\begin{array}{l}\text { Exchange rates on } 31.12 .98 \\
\text { DM / } 2.7980 \\
\text { DM /Sfr } 122.20 \\
\text { DM / PTS } 1.1755 \\
\text { DM / ATS } 14.214\end{array}$ & \multicolumn{2}{|c|}{$\begin{array}{l}\text { Exchange rates on: } 31.12 .99 \\
\text { f/Euro: } 1,61060 \\
\text { SFr/Euro: } 0,62330 \\
\text { PTS/Euro (100): } 0,60100 \\
\text { DM/Euro: } 0,51130\end{array}$} \\
\hline
\end{tabular}


Table 2. Future EASD Annual Meetings

\begin{tabular}{lll}
\hline 2002 & $1-5$ September & Budapest, Hungary \\
2003 & $24-29$ August & $18^{\text {th }}$ IDF Congress, Paris, France \\
2004 & & Munich, Germany \\
2005 & & Athens, Greece \\
2006 & Copenhagen/Malmö, Denmark/Sweden \\
2007 & Amsterdam, The Netherlands \\
2008 & Torino, Italy \\
\hline
\end{tabular}

Table 3. Breakdown of membership by country compared to previous years (as of $31^{\text {st }}$ December):

\begin{tabular}{|c|c|c|c|c|c|c|c|c|c|c|c|}
\hline \multirow[b]{2}{*}{ Albania } & \multicolumn{11}{|c|}{19991998199719961995199419931992199119901989} \\
\hline & 4 & 1 & 1 & 1 & & & & & & & \\
\hline Algeria & 8 & 8 & 6 & 13 & 2 & 4 & 4 & 1 & - & 1 & 1 \\
\hline & 12 & 12 & 9 & 8 & 7 & 8 & & 6 & 6 & 6 & 8 \\
\hline & 1 & 2 & 2 & 1 & - & - & - & - & - & - & - \\
\hline & 87 & 97 & 66 & 79 & 69 & 62 & 66 & 61 & 54 & 52 & 48 \\
\hline & 93 & 85 & 71 & 112 & 70 & 60 & 54 & 57 & 37 & 34 & 39 \\
\hline Se & 5 & 9 & 10 & 7 & & 7 & - & - & - & - & - \\
\hline & 1 & 2 & - & 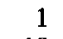 & 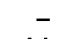 & - & - & - & - & - & - \\
\hline des & 13 & 28 & 23 & 18 & 14 & 6 & 3 & 3 & 1 & 1 & 1 \\
\hline B & 185 & 145 & 122 & 153 & 144 & 133 & 154 & 161 . & 140 & 138 & 140 \\
\hline & - & - & - & - & 1 & 1 & - & - & - & - & - \\
\hline \multicolumn{12}{|l|}{ Bosnia- } \\
\hline Herzegov & 19 & 12 & 3 & & & & & & & & \\
\hline & 28 & 32 & 19 & 20 & 13 & 12 & 10 & 9 & 9 & 9 & 9 \\
\hline & 41 & 41 & 34 & 66 & 62 & 67 & 59 & 36 & 32 & 24 & 19 \\
\hline & 13 & - & 7 & 7 & 2 & 4 & 1 & - & 1 & - & - \\
\hline & 1 & - & 1 & 1 & - & - & - & - & - & - & - \\
\hline & 39 & 47 & 29 & 34 & 38 & 37 & 36 & 32 & 33 & 33 & 35 \\
\hline & 1 & 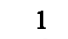 & 1 & 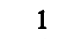 & 1 & - & - & - & - & - & - \\
\hline & 6 & 6 & 4 & 4 & 5 & 4 & 3 & 3 & 4 & 4 & 4 \\
\hline & 7 & 5 & 2 & 1 & 1 & 2 & 1 & 1 & 2 & 2 & 2 \\
\hline & 2 & 2 & 3 & 3 & 2 & 2 & 1 & 1 & - & 1 & 1 \\
\hline & - & - & - & - & - & 1 & - & 1 & 1 & 1 & 1 \\
\hline & 71 & 48 & 44 & 35 & 33 & 38 & 25 & 22 & 25 & - & - \\
\hline & 3 & . & $\because$ & 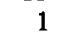 & & 2 & & - & - & - & 1 \\
\hline & 10 & 9 & 9 & 8 & $f$ & 9 & 8 & 8 & 8 & 10 & 6 \\
\hline & 49 & 58 & 45 & 37 & 51 & 60 & 44 & 45 & 70 & 68 & 52 \\
\hline & 303 & 327 & 241 & 284 & 275 & 249 & 273 & 266 & 256 & 267 & 206 \\
\hline \multicolumn{12}{|l|}{ Dominican } \\
\hline Pen & 1 & 1 & 1 & 1 & 2 & 2 & 1 & 1 & - & 1 & 1 \\
\hline & 3 & 3 & 1 & - & 1 & 1 & - & - & - & - & - \\
\hline & 29 & 27 & 19 & 23 & 23 & 20 & 11 & 12 & 12 & 13 & 9 \\
\hline & 30 & 19 & 19 & 17 & 17 & 8 & 7 & 6 & 5 & - & - \\
\hline & 136 & 125 & 91 & 111 & 2 & 96 & 93 & 00 & 95 & 97 & 87 \\
\hline & 91 & & 53 & 216 & 29 & 205 & 17 & 218 & 222 & 252 & 254 \\
\hline & 7 & & 7 & 5 & 3 & 2 & 2 & 1 & 1 & - & - \\
\hline & 559 & 515 & 440 & 488 & 435 & 416 & 363 & 350 & 315 & 319 & 262 \\
\hline & 150 & 156 & 141 & 138 & 145 & 138 & 126 & 125 & 131 & 118 & 122 \\
\hline G & - & - & - & - & 1 & 1 & - & - & - & - & - \\
\hline & - & - & - & 1 & 1 & 1 & - & - & - & - & - \\
\hline & - & - & - & 1 & - & - & - & - & - & - & - \\
\hline & 90 & 85 & 63 & 95 & 62 & 63 & 58 & 56 & 48 & 42 & 41 \\
\hline & 2 & 1 & 1 & 1 & - & - & - & - & - & - & - \\
\hline & 19 & 14 & 11 & 14 & 14 & 9 & 8 & 9 & 6 & 7 & 8 \\
\hline & 4 & - & 5 & 3 & 1 & 1 & 1 & 1 & 1 & - & 2 \\
\hline & 2 & 1 & - & - & & - & - & - & - & - & - \\
\hline & 2 & 1 & 1 & 2 & 1 & 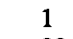 & 1 & 1 & - & - & - \\
\hline & 30 & 24 & 16 & 18 & 20 & 23 & 29 & 34 & 38 & 22 & 14 \\
\hline & 55 & 65 & 44 & 50 & 46 & 55 & 48 & 35 & 40 & 45 & 45 \\
\hline & 284 & $334^{\circ}$ & 274 & 311 & 373 & 451 & 422 & 454 & 427 & 390 & 317 \\
\hline & 173 & 162 & 136 & 141 & 112 & 86 & 76 & 62 & 52 & 49 & 40 \\
\hline & 6 & 6 & 1 & 3 & - & - & - & - & - & - & - \\
\hline 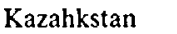 & - & 3 & 3 & - & - & 2 & 3 & 3 & 1 & - & - \\
\hline & 1 & - & - & 2 & - & - & 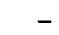 & - & - & - & 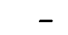 \\
\hline 14 & - & 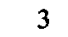 & & 1 & - & - & - & - & - & - & \\
\hline & 11 & 7 & 5 & 2 & & & & 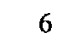 & 3 & 11 & 4 \\
\hline & 3 & 3 & 4 & 7 & 3 & 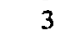 & 3 & 3 & 4 & 5 & 4 \\
\hline & 11 & 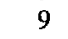 & 4 & 7 & 6 & 5 & 1 & 1 & - & - & - \\
\hline Le & 1 & 1 & 1 & 2 & 2 & 1 & 1 & 1 & 1 & 1 & 1 \\
\hline Libya & 1 & 1 & - & - & - & - & - & - & - & - & 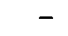 \\
\hline
\end{tabular}

\begin{tabular}{|c|c|c|c|c|c|c|c|c|c|c|c|c|}
\hline & 199 & 1998 & 1997 & 199 & 6 199: & 51994 & 1993 & 1992 & 21991 & 11990 & 0198 & \\
\hline Lithuania & 22 & 25 & 21 & 14 & 12 & 12 & 10 & 9 & 9 & - & & \\
\hline Luxemburg & 5 & 5 & 5 & 5 & 5 & 5 & 3 & 3 & 2 & 2 & 1 & 1 \\
\hline Macao & - & 1 & 1 & 1 & 1 & 1 & 1 & 1 & - & - & & - \\
\hline FYRoMface- & & & & & & & & & & & & \\
\hline donia & 11 & 16 & 16 & 9 & 12 & 11 & 7 & 2 & - & - & & - \\
\hline Malaysia & - & - & 1 & - & 1 & - & - & - & - & - & & - \\
\hline Malta & 1 & 1 & 1 & 4 & 3 & 3 & 4 & - & - & - & & - \\
\hline Mauritius & - & - & 1 & 1 & - & - & - & - & - & - & & - \\
\hline Mexico & 8 & 7 & 4 & 3 & 4 & 4 & 3 & 3 & 3 & 2 & & 2 \\
\hline Moldavia & 3 & 3 & 2 & & & & & & & & & \\
\hline Morocco & 5 & 5 & 5 & 5 & 1 & 2 & 2 & 2 & 3 & 3 & & 3 \\
\hline New Zealand & 15 & 13 & 10 & 11 & 9 & 8 & 10 & 10 & 11 & 7 & & 5 \\
\hline Norway & 81 & 76 & 59 & 77 & 102 & 70 & 105 & 76 & 68 & 72 & 62 & \\
\hline Oman & 1 & 1 & 2 & 3 & 2 & 1 & 1 & 1 & 1 & 1 & & 1 \\
\hline Pakistan & 9 & 9 & 4 & 6 & 6 & 5 & 3 & 2 & 2 & 2 & & 1 \\
\hline Panama & - & - & - & - & 1 & 1 & - & - & - & - & & - \\
\hline Pap.New & & & & & & & & & & & & \\
\hline Guinea & - & 1 & - & - & - & - & - & - & - & - & & - \\
\hline Paraguay & 3 & 5 & 4 & 3 & 2 & 2 & 1 & 1 & 1 & 1 & & 1 \\
\hline Peru & 1 & 1 & - & 1 & 2 & 2 & - & 1 & 1 & 2 & & 2 \\
\hline Philippines & 2 & 2 & 2 & 2 & 1 & 1 & 1 & 1 & 2 & 3 & & - \\
\hline Poland & 146 & 88 & 79 & 93 & 93 & 105 & 80 & 63 & 56 & 42 & 3 & 1 \\
\hline Portugal & 83 & 85 & 68 & 103 & 97 & 97 & 105 & 55 & 57 & 54 & 5 & \\
\hline Puerto Rico & - & - & - & - & - & 1 & 1 & - & - & - & & - \\
\hline Qatar & - & - & - & 1 & 1 & 1 & - & - & - & 1 & & 1 \\
\hline Reunion & 2 & - & - & 2 & - & - & - & - & - & - & & - \\
\hline Romania & 94 & 68 & 55 & 54 & 48 & 51 & 44 & 38 & 59 & 49 & 4 & \\
\hline Russia & 82 & 84 & 61 & 49 & 56 & 52 & 37 & 35 & 30 & - & & - \\
\hline Saudi Arabia & 9 & 10 & 6 & 12 & 8 & 14 & 17 & 19 & 9 & 8 & & 9 \\
\hline Singapore & 6 & 4 & 3 & 3 & 4 & 4 & 3 & 3 & 4 & 4 & & - \\
\hline Slovakia & 31 & 37 & 23 & 32 & 32 & 27 & 20 & - & - & - & & - \\
\hline Slovenia & 53 & 54 & 40 & 40 & 30 & 30 & 21 & 13 & 7 & - & & - \\
\hline South Africa & 25 & 17 & 16 & 22 & 14 & 26 & 27 & 35 & 27 & 29 & 1 & \\
\hline Spain & 240 & 374 & 174 & 226 & 199 & 172 & 162 & 152 & 131 & 122 & 10 & \\
\hline Sri Lanka & - & 1 & - & 1 & 1 & 1 & 1 & 1 & 1 & - & & - \\
\hline Sudan & 1 & - & - & 1 & 1 & 1 & 1 & 1 & 1 & 1 & & 1 \\
\hline Sweden & 295 & 334 & 250 & 342 & 353 & 250 & 310 & 337 & 270 & 253 & 26 & \\
\hline Switzerland & 94 & 91 & 79 & 83 & 75 & 79 & 86 & 85 & 10.4 & 110 & 11 & \\
\hline Syria & 1 & 1 & 1 & & & & & & & & & \\
\hline Tahiti & - & 1 & 1 & 1 & 1 & 1 & 1 & - & - & - & & - \\
\hline Taiwan & 10 & 10 & 8 & 11 & 10 & 10 & 12 & 8 & 7 & 6 & & 4 \\
\hline Tanzania & 1 & 2 & 1 & 2 & 2 & 2 & 2 & 1 & - & 1 & & 1 \\
\hline Tatarstan & - & - & - & - & 1 & 1 & 1 & 1 & - & - & & - \\
\hline Thailand & 17 & 9 & 4 & 4 & 4 & 4 & 4 & 4 & 3 & 3 & & 3 \\
\hline $\begin{array}{l}\text { The Nether- } \\
\text { lands }\end{array}$ & 222 & 244 & 170 & 212 & 232 & 245 & 213 & 180 & 151 & 150 & 14 & \\
\hline Tonga & - & - & - & - & - & 1 & 1 & 1 & - & - & & - \\
\hline Tunisia & 1 & 2 & 2 & 2 & 2 & 2 & 1 & 1 & 1 & - & & - \\
\hline Turkey & 49 & 45 & 42 & 46 & 38 & 42 & 32 & 21 & 13 & 11 & 1 & 11 \\
\hline Ukraine & 62 & 45 & 26 & 27 & 34 & 29 & 16 & 14 & 10 & - & & - \\
\hline United A & 1 & 1 & - & 2 & 1 & 2 & 3 & 1 & 2 & 2 & & 1 \\
\hline Emirates & & & & & & & & & & & & \\
\hline United & & & & & & & & & & & & \\
\hline Kingdom & 484 & 515 & 404 & 461 & 430 & 390 & 404 & 396 & 495 & 420 & 40 & \\
\hline USA & 276 & 301 & 263 & 276 & 264 & 239 & 232 & 209 & 199 & 169 & 16 & \\
\hline Uruguay & 1 & 2 & 1 & - & 1 & 1 & - & - & - & - & & - \\
\hline Uzbekistan & 4 & 5 & 4 & 4 & 1 & 1 & 1 & 1 & 1 & 1 & & 1 \\
\hline Venezuela & 1 & - & - & - & 1 & 1 & - & - & - & - & & - \\
\hline Yugoslavia & 40 & 36 & 36 & 31 & 35 & 32 & - & 15 & 18 & 37 & 3 & 30 \\
\hline Zimbabwe & 1 & 1 & 1 & 1 & 1 & 1 & 1 & 1 & 1 & 1 & & 1 \\
\hline
\end{tabular}

Table 4. Figures for membership at the end of 1999 compared to previous years:

199919981997199619951994199319921991199019891988198719861985 $53035379416+485446504406424440003847364533172918231721811839$ 